\title{
Pre-neoplastic lesion, mucin-depleted foci, reveals de novo high-grade dysplasia in rat colon carcinogenesis
}

\author{
CHANGXU CUI ${ }^{1,2}$, REIKA TAKAMATSU ${ }^{1}$, HIROSHI DOGUCHI ${ }^{1}$, \\ AKIKO MATSUZAKI $^{1}$, MASANAO SAIO ${ }^{1}$ and NAOKI YOSHIMI ${ }^{1}$ \\ ${ }^{1}$ Department of Pathology and Oncology, Graduate School of Medical Science, University of the Ryukyus, Okinawa, Japan; \\ ${ }^{2}$ Department of Biochemistry and Molecular Biology, Yanbian University Health Science Center, Yanji, P.R. China
}

Received November 9, 2011; Accepted December 23, 2011

DOI: $10.3892 /$ or.2012.1657

\begin{abstract}
Aberrant crypt foci (ACF) and mucin-depleted foci (MDF) have recently been recognized as pre-neoplastic lesions in the colon of carcinogen-treated rodents. In the present study, we analyzed the sequential development of ACF and MDF histopathologically in the colon of rats from 5 to 40 weeks after DMH treatment. The numbers of ACF per colon increased over time during the experiment, and were much higher than the number in tumors, while the number of MDF per colon remained unchanged from the early stage (the 5th week after carcinogen exposure), and approximate to those in tumors. The incidence of ACF, which was much higher than that of tumors, also increased gradually in a time-dependent manner. The incidence of MDF, however, was similar to that of tumors and did not change significantly during the whole experiment. No lesion as dysplasia with high-grade (DHG) or adenocarcinoma (AC) were found in any large ACF from the 5th to 40th week histopathologically, whereas all of the large MDF showed DHG or AC features. Even at 5 weeks, MDF showed features of DHG. We classified these into two forms of MDF: flat and protruded MDF. At 40 weeks, the number of flat MDF per colon decreased significantly compared with that at 20 weeks $(\mathrm{p}<0.05)$, however, the number of protruded MDF per colon increased $(\mathrm{p}<0.01)$, and the percentage of DHG in a protruded MDF lesion decreased but that of $\mathrm{AC}$ increased remarkably. In conclusion, MDF may develop into cancer through the so-called 'de novo cancer' pathway.
\end{abstract}

Correspondence to: Dr Changxu Cui, Department of Pathology and Oncology, Graduate School of Medical Science, University of the Ryukyus, Nishihara, Okinawa 903-0125, Japan

E-mail: xu9659@mail.ryudai.jp

Abbreviations: ACF, aberrant crypt foci; MDF, mucin-depleted foci; DMH, 1,2-dimethylhydrazine dihydrochloride; BCAC, $\beta$-catenin accumulated crypts; HPLL, hyperplastic polyp-like lesion; DLG, dysplasia (intraepithelial) with low-grade; DHG, dysplasia (intraepithelial) with high-grade; AC, adenocarcinoma

Key words: colon carcinogenesis, pre-neoplastic lesions, mucindepleted foci

\section{Introduction}

Colon carcinogenesis occurs through consecutive steps, from normal crypt into pre-neoplastic lesion, adenoma, and finally change into carcinoma (1). In this process, the formation of early neoplastic lesions with various degrees of dysplasia is a crucial point. Accordingly, many efforts have been devoted to the identification and characterization of such lesions (2-5). Aberrant crypt foci (ACF), which was initially identified topographically on the colonic mucosa of rodents exposed to colorectal carcinogens, have been believed as a pre-neoplastic lesion in many studies of chemoprevention. Actually, the simple identification of ACF in experiments is very useful and important as a biomarker, because it does not need histopathological knowledge. Furthermore, a similar lesion has been also observed in humans. The association between ACF and development of colonic neoplasia has been described both in rodents and human $(2,4,6,7)$. In contrast, there are several studies, which show lack of association between ACF and development of colonic neoplasms (8-10).

Previously, we have proposed $\beta$-catenin accumulated crypts (BCAC) as one of more predictive biomarkers in rat colon carcinogenesis $(11,12)$. However, the identification require histological steps and immunohistochemistry. Recently, mucin-depleted foci (MDF) have been described to be the pre-neoplastic lesion which could be used as biomarkers in colon carcinogenesis. MDF were also identified in colon mucosa at high risk of colon cancer in human $(8,13,14)$. The identification of MDF is relatively simple and examined topographically on the colonic mucosa with mucin staining instead of methylene bleu staining as well as ACF. We also reported that MDF has similar characteristics to BCAC rather than ACF (15), but there are no reports on the sequential histopathological analyses of MDF to our knowledge.

In the present study we examined the sequential development of ACF and MDF in the colon of rats from 5 to 40 weeks after DMH treatment. In order to identify early lesions with a morphological and developmental relationship with tumorigenesis, we classified the developing lesions by surface examination, quantified them, determined their growth and examined them sequentially and histopathologically. 


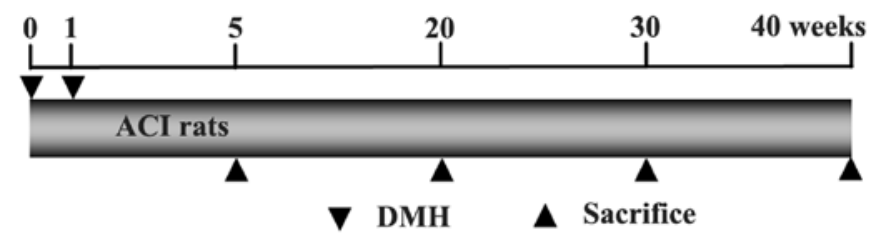

Figure 1. Experimental design. The rats were treated by subcutaneous injection of DMH with a dose of $40 \mathrm{mg} / \mathrm{kg}$ body weight, once a week for 2 weeks. At different times after DMH (arrows) rats were sacrificed.

\section{Materials and methods}

Animals and treatments. Four-week-old male ACI rats were obtained from Japan SLC, Inc. (Hamamatsu, Japan). All animals were housed in wire cages (3 rats/cage) with free access to drinking water and a basal diet, CE-2 (CLEA Japan, Inc., Tokyo), under controlled conditions of humidity $(50 \pm 10 \%)$, lighting (12-h light/dark cycle) and temperature $\left(23 \pm 2^{\circ} \mathrm{C}\right) . \mathrm{DMH}$ was purchased from Sigma-Aldrich Chemical Co. (St. Louis, MO). This study was approved by the Animal Welfare Committee of the University of the Ryukyus.

Experimental protocol. Experimental protocol is shown in Fig. 1. In brief, after quarantine for 1 week, the experiment was started at 5 weeks of age, the rats were treated by subcutaneous injection of DMH with a dose of $40 \mathrm{mg} / \mathrm{kg}$ body weight, once a week for 2 weeks. At 5, 20, 30 and 40 weeks after the first DMH treatment, rats were sacrificed under $\mathrm{CO}_{2}$ anesthesia. Immediately after sacrifice, the colons were removed, and fixed in $10 \%$ buffered formalin.

Identification of ACF and MDF in unsectioned colon. Alcian blue staining was used to identify both ACF and MDF, instead of the conventional staining using $0.2 \%$ methylene blue and high-iron diamine Alcian blue, respectively $(2,8)$. Briefly, the fixed colons were rinsed for $5 \mathrm{~min}$ in $3 \%$ acetic acid and then stained for $5 \mathrm{~min}$ with $1 \%$ Alcian blue solution ( $\mathrm{pH} 2.5)$ in $3 \%$ acetic acid. These colons were rinsed for $5 \mathrm{~min}$ in $3 \%$ acetic acid to prevent non-specific staining and then washed in distilled water (15). ACF were identified according to the following criteria: larger than the adjacent normal crypts and elevated with thickened cell wall lining the crypt and increased pericryptal area and multiplicity (i.e., the number of crypts forming each focus) higher than 4 crypts (Fig. 2A), in accordance with other studies using methylene blue staining (2), in addition to crypts with mucous production. MDF were identified as focal lesions by the following criteria: i) absence or very small production of mucins; ii) distortion of the opening of the lumen compared with normal surrounding crypts; iii) multiplicity higher than 4 crypts $(8,15)$. MDF can be divided into two types according to morphological characteristics. One is flat paralleled with normal mucosa; the other is protruded as crypts expanding and swelling over normal mucosa. Both types are judged as negative in Alcian blue staining. The latter looks like ACF in conventional methylene blue staining, it was described as overlapping lesion in a previous study (15). We herewith classified and counted MDF with a flat lesion (a flat MDF,
Fig. 2B) and MDF with a protruded change (a protruded MDF, Fig. 2C), respectively. The stained mucosa including both ACF and MDF were photographed with a DP-50 digital camera (Olympus Optical Co., Ltd., Tokyo) and the positions of both lesions were marked on the captured images in a computer monitor. The numbers of MDF and ACF per colon were recorded. In addition, some ACF was also confirmed by conventional methylene blue staining.

Histopathological examinations. After the identification of ACF and MDF, they were embedded in paraffin for histological analyses. Colonic mucosal sections were examined by utilizing both an en face preparation (11) and a conventional vertical preparation for a half section of each lesion, respectively. Sections were stained with hematoxylin and eosin (H\&E). The computer-captured images including ACF and MDF in unsectioned colons were compared with the histological lesions in the sections. The histopathological diagnoses of the lesions were defined as four types: i) hyperplastic polyp-like lesion (HPLL); ii) dysplasia (intraepithelial neoplasia) with low-grade (DLG); iii) dysplasia (intraepithelial neoplasia) with high-grade (DHG); iv) adenocarcinoma (AC), according to the criteria (16-19) with our modification by decrease or loss of goblet cells, the crypt structure, and the nuclear abnormalities (Fig. 3).

Statistical analysis. Data are presented as mean \pm SE. Student's t-test was used to determine the significance of differences between groups. P-values $<0.05$ were considered to be significant.

\section{Results}

Identification of $A C F$ and $M D F$. Although numbers of $\mathrm{ACF}$ per colon were increased in a time-dependent manner, those of MDF including flat and protruded types kept relatively stable. At 20 weeks, the numbers of ACF and MDF were 24.17 \pm 2.1 and $0.5 \pm 0.15$, respectively. Although the numbers of ACF showed significantly higher than those in tumors at 40 weeks $(0.8 \pm 0.27)$, those of MDF were not significantly different from those of tumors, i.e. $>3 \mathrm{~mm}$ in size macroscopically at sacrifice (Fig. 4A and B). Incidence of ACF gradually increased together with time, while that of MDF was relatively stable. At 20 weeks, the incidence of ACF and MDF were 100 and $50 \%$, respectively. At 40 weeks, the incidence of MDF was approximate to that in tumors (45\%) (Fig. 4C). Then we evaluated the time-dependent changes of the numbers of both flat and protruded MDF with more than 4 crypts. The numbers per colon and incidence of both flat and protruded MDF were stable until 20 weeks (Fig. 5A and B). At 40 weeks, the end of the experiment, the numbers of a flat MDF showed a significant reduction $(\mathrm{p}<0.05)$, while those of a protruded MDF were up-regulated $(\mathrm{p}<0.01)$.

Histopathological features corresponding to ACF and MDF. Representative micro-stereoscopic and histological findings of ACF and MDF within 4 crypts in early stage (Fig. 6A-F) and with more than 10 crypts in late stages (Fig. 6G-L) was demonstrated. ACF consisted of HPLL (54.5\%) and DLG $(45.5 \%)$ histopathologically, while in MDF, $62.1 \%$ was DHG 


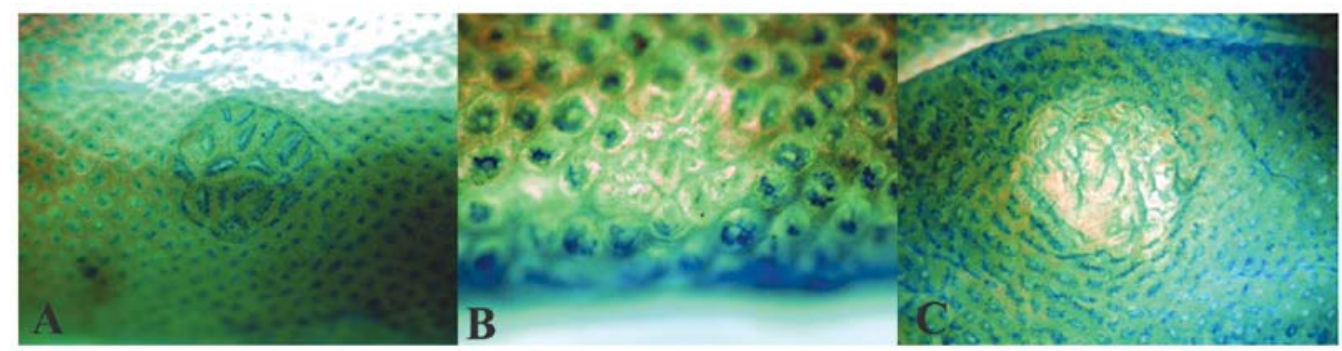

Figure 2. Morphological appearance of each lesion in the rat colon stained with Alcian blue staining. (A) ACF; (B) a flat MDF; (C) a protruded MDF. Original magnification, $\mathrm{x} 40$ in ACF and protruded MDF; $\mathrm{x} 100$ in MDF.

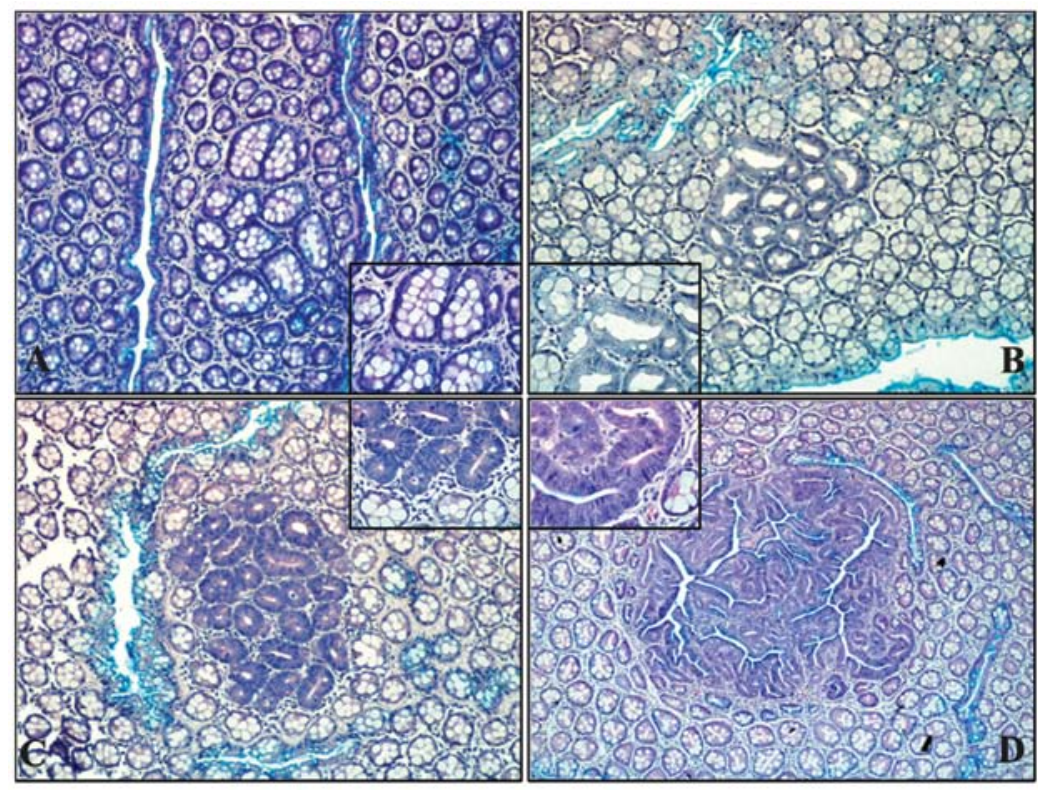

Figure 3. Histopathological findings of each classification of the pre-neoplastic or neoplastic lesions. (A) Hyperplastic polyp-like lesion; (B) dysplasia (intraepithelial), low grade; (C) dysplasia (intraepithelial), high grade; (D) adenocarcinoma. Original magnification, x100.
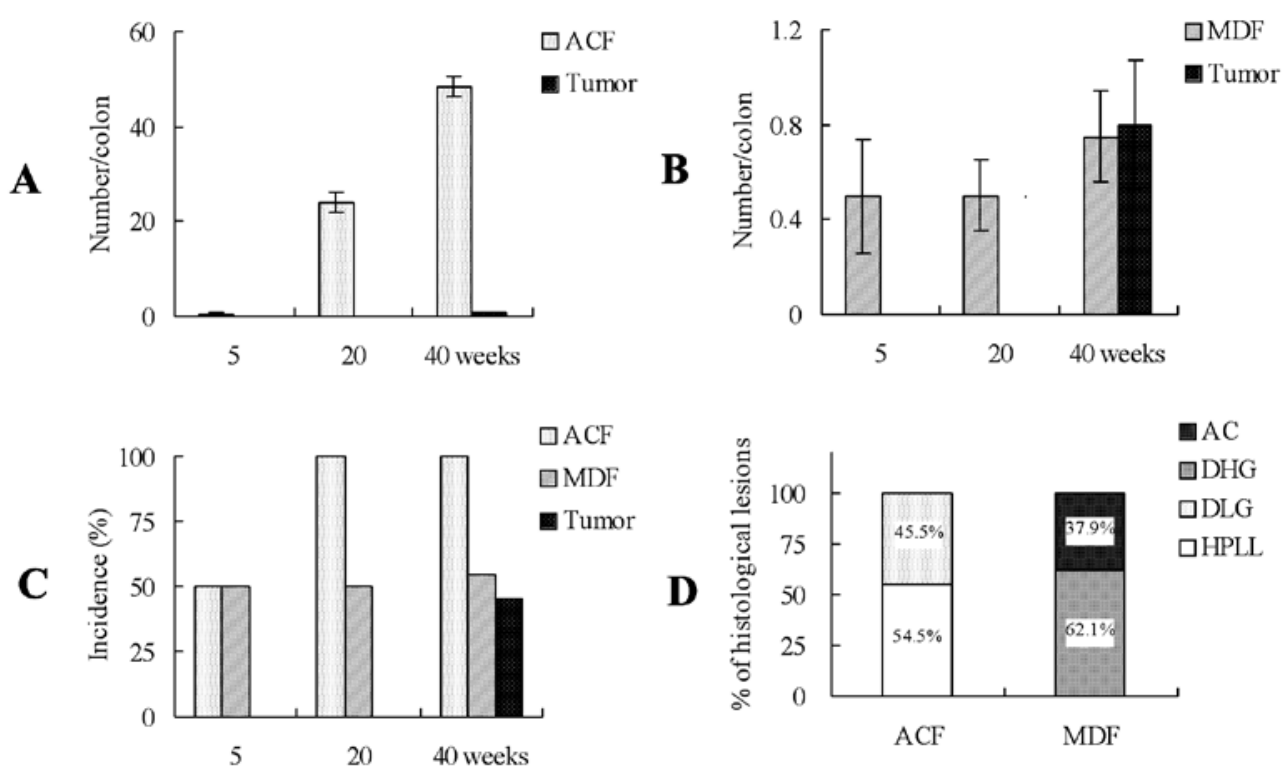

Figure 4. The sequential development of ACF and MDF in the colon of rats from 5 to 40 weeks after DMH treatment. (A) The number of ACF per colon increased over time during the experiment, and was much more than the number in tumors; (B) the number of MDF per colon remained unchanged from the early stage, and approximate to that in the tumor; $(C)$ the incidence of $\mathrm{ACF}$, which was much higher than that in the tumor. The incidence of MDF was similar to that in the tumor during the whole experiment; (D) no DHG was found in any large ACF from the 5th to 40th week in the experiment, whereas all of the large MDFs showed DHG or adenocarcinoma. Values represent mean \pm SE. 

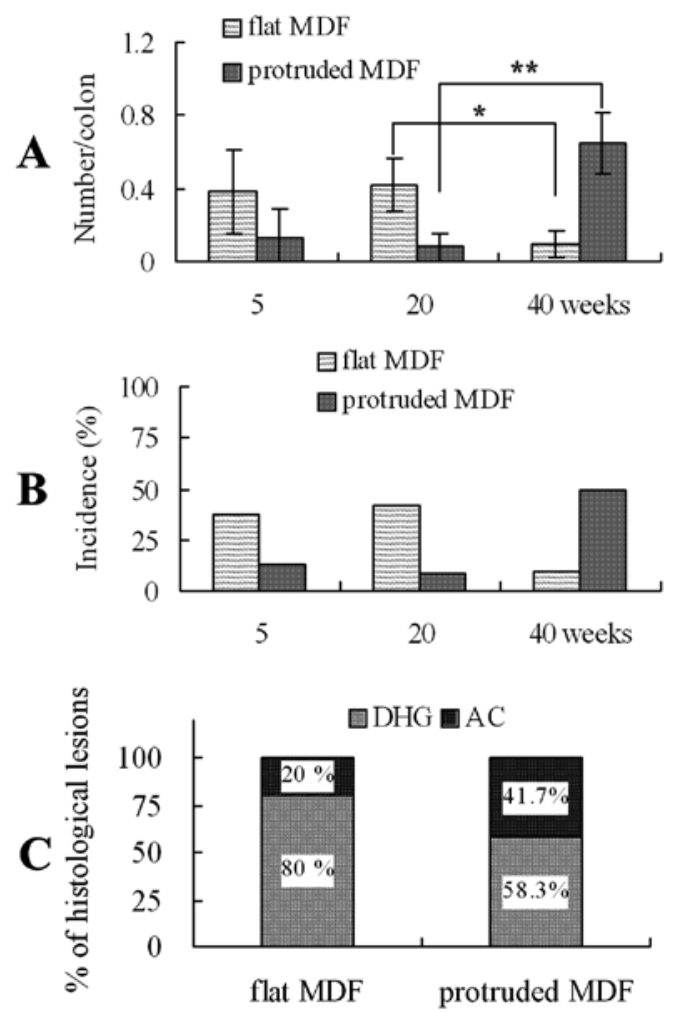

Figure 5. The sequential development of flat MDF and protruded MDF in the colon of rats from 5 to 40 weeks after DMH treatment. (A) In the 40 weeks, number of a flat MDF per colon decreased significantly compared with that in the 20 weeks, but number of a protruded MDF per colon increased; (B) at the level of incidence, flat MDF and protruded MDF was also similar to process of changes in number; $(C)$ in histopathology, in contrast with flat MDF, the percentage of DHG in a protruded MDF decreased but that of AC increased remarkably. Values represent mean $\pm \mathrm{SE}$. ${ }^{*} \mathrm{p}<0.05$, ${ }^{* *} \mathrm{p}<0.01$

and $37.9 \%$ was AC. In the ACF, there was no malignancy. All MDF have malignant potential (Fig. 4D). Then we analyzed the histopathological difference between flat and protruded MDF. In a flat MDF, $80 \%$ was DHG, $20 \%$ was $\mathrm{AC}$ and $58.3 \%$ was DHG, while $41.7 \%$ was AC in a protruded MDF. The percentage of DHG in a protruded MDF was obviously lower than that in a flat MDF. In contrast, the percentage of AC in a protruded MDF was increased compared with that in a flat MDF (Fig. 5C).

\section{Discussion}

In this study, we observed the sequential and histopathological changes of MDF in detail, which is reported as one of useful biomarkers in animal colon carcinogenesis by Caderni et al (8). It showed that the numbers of ACF per colon increased together with the experimental duration, while those of MDF per colon were unchanged from early stage (at 5 weeks after carcinogen exposure) (Fig. 4A and B). The counted numbers of ACF were similar to the results of a previous study (20). Furthermore, the incidence of ACF also increased gradually in a time-dependent manner, but that of MDF did not significantly change (Fig. 4C). By histological examination, no DHG was found in any large $\mathrm{ACF}$ from the 5th to 40th week in the experiment, whereas all of the large MDF showed DHG or adenocarcinoma (Fig. 4D).

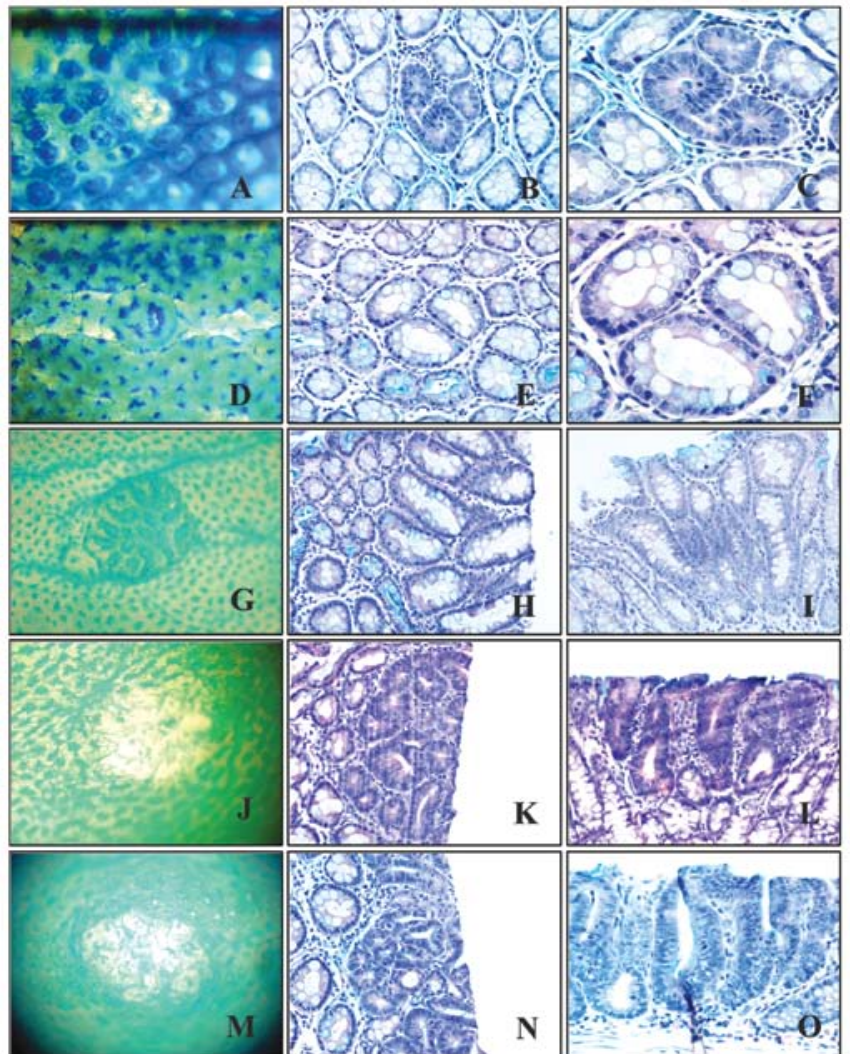

Figure 6. Representative features of the examined small lesions at 5 week (A-F). (A-C) MDF in AB staining (A) the corresponding H\&E-stained lesion (B and C); (D-F) ACF in AB staining (D) the corresponding H\&E-stained lesion ( $\mathrm{E}$ and $\mathrm{F}$ ). Representative of the examined large lesions (more than 10 crypts) at late stage in AB staining the corresponding H\&E-stained lesion by en face preparation and a vertical preparation, respectively (G-O). (G-I) ACF; (J-L) a flat MDF; (M-O) a protruded MDF.

Hence, our data suggest that MDF are histopathologically associated with more malignant potential than ACF.

MDF has been identified along the entire mucosal surface of unbedded colon as well as ACF (8). It is essential and important that MDF is related to colonic mucins. Mucins are highly glycosylated proteins that are the major components of the mucins that lubricate and protect the underlying intestinal epithelium (21). Although alterations of mucin expression and glycosylation have been observed in human colon cancer specimens (22), the role of these changes in the development of tumor has yet to be clarified. Moreover, it has been noted that sialomucin increased relatively due to a reduction of sulfomucin in colon cancer in human and rats (23-27). Therefore, Caderni et al used high-iron diamine Alcian blue staining, which can stain for sialomucin and sulfomucin respectively, for the detection of MDF (8). However, high-iron diamine Alcian blue staining is not convenient compared with methylene blue staining for ACF, and we used simple Alcian blue (pH 2.5) staining for MDF as described in our previous study (15), because a simple approach for the identification of mucin depletion might largely contribute to detect early malignancy.

Concerning ACF, there are several ACF such as classical and dysplastic ACF (28). Paulsen et al reported that the lesion defined as a flat $\mathrm{ACF}$, which is recognized with methylene blue staining, seems like a useful biomarker in animal colon carci- 
nogenesis (29,30). It looks like MDF, and actually, Femia et al collaborated to examine both lesions (31). In their study, over a half of both lesions are identical. Ochiai et al also defined a flat and dysplastic ACF by methylene blue staining $(28,32)$. However, it is difficult and complex to compare the description of ACF lesions such as Paulsen and Ochiai, because the staining time and the staining patterns between the studies are quite different. Therefore, the same nomenclature as ACF might be confused. ACF, observed in this study, also is slightly different from the original $\mathrm{ACF}$, because of our identification of $\mathrm{ACF}$ is dependent on the tissues with $\mathrm{AB}$ staining as described in Materials in methods. The most important issue in this study is to decrease the mucin of pre-neoplastic or neoplastic lesions as MDF and represent the characteristics of histological findings.

In this study, we have proposed a new classification of MDF such as a flat MDF and a protruded MDF. The latter, which we reported as overlapped lesion in our previous study (15), looks like topographical ACF appearance on the colonic mucosa. When we analyzed the two forms of MDF, such as a flat and a protruded MDF, numbers of both flat and protruded MDF per colon did not change by the end of 20th week. At 40 weeks, however, with formation of tumor, number of flat MDF per colon decreased significantly compared with that at 20 weeks, but the number of protruded MDF per colon increased (Fig. 5A). In histopathology, in contrast with flat $\mathrm{MDF}$, the percentage of DHG in a protruded MDF decreased but that of AC increased remarkably (Fig. 5C). Our findings suggest that a flat MDF may transform into a protruded MDF gradually with time.

In addition, histopathological findings of MDF or BCAC at 5 weeks revealed high-grade adenoma with severe nuclear atypia (12,15), as well as those of Fig. 6B and $\mathrm{C}$ in this study. In this study, we diagnosed it as DHG according to the WHO classification (18). Our findings indicated that all of the small MDFs (less than 4 crypts) showed DHG histopathologically, and no $\mathrm{AC}$ was found in the early stage. But over time, AC appeared in the large MDFs (more than 10 crypts) during the late stage (Figs. 4D and 6K and L). As the differential diagnosis between adenoma and adenocarcinoma is usually done by the invasive characteristics $(18,19)$, we diagnosed the tumors according to these criteria. However, in humans, the existence of 'intramucosal carcinoma' has been recognized indicating non-invasive cancer (18). In fact, many Japanese surgical/anatomic pathologists may recognize them as the feature of carcinoma in situ. This is a well known difference in the diagnosis of tumor of the gastrointestinal tract in humans between Western countries and Japan (33). According to Japanese criteria for colonic tumors, small MDF is close to the malignancy and de novo pathway in colon carcinogenesis.

In conclusion, MDF may develop into cancer through the so-called 'de novo cancer' pathway histopathologically. However, it was difficult to distinguish large (dysplastic) ACF from protruded MDF even in this study. Further research on molecular changes in a protruded $\mathrm{MDF}$, concerning 'de novo cancer' is required.

\section{Acknowledgements}

This study was supported in part by a Grant-in-Aid from the Ministry of Education, Culture, Sports, Science and
Technology of Japan and a Grant-in-Aid from the Ministry of Health, Labour and Welfare of Japan. We also acknowledge all members of our laboratories for the helpful comments and collaborations, especially Eriko Miyagi.

\section{References}

1. Chang WW: Histogenesis of colon cancer in experimental animals. Scand J Gastroenterol (Suppl) 104: 27-43, 1984.

2. Bird RP: Observation and quantification of aberrant crypts in the murine colon treated with a colon carcinogen: preliminary findings. Cancer Lett 37: 147-151, 1987.

3. McLellan EA, Medline A and Bird RP: Sequential analyses of the growth and morphological characteristics of aberrant crypt foci: putative preneoplastic lesions. Cancer Res 51: 5270-5274, 1991.

4. Pretlow TP, Barrow BJ, Ashton WS, et al: Aberrant crypts: putative preneoplastic foci in human colonic mucosa. Cancer Res 51: 1564-1567, 1991.

5. Roncucci L, Stamp D, Medline A, Cullen JB and Bruce WR: Identification and quantification of aberrant crypt foci and micro-adenomas in the human colon. Hum Pathol 22: 287-294, 1991.

6. Bird RP, McLellan EA and Bruce WR: Aberrant crypts, putative precancerous lesions, in the study of the role of diet in the aetiology of colon cancer. Cancer Surv 8: 189-200, 1989.

7. Fenoglio-Preiser CM and Noffsinger A: Aberrant crypt foci: a review. Toxicol Pathol 27: 632-642, 1999.

8. Caderni G, Femia AP, Giannini A, et al: Identification of mucindepleted foci in the unsectioned colon of azoxymethane-treated rats: correlation with carcinogenesis. Cancer Res 63: 2388-2392, 2003.

9. Magnuson BA, Carr I and Bird RP: Ability of aberrant crypt foci characteristics to predict colonic tumor incidence in rats fed cholic acid. Cancer Res 53: 4499-4504, 1993.

10. Zheng Y, Kramer PM, Lubet RA, Steele VE, Kelloff GJ and Pereira MA: Effect of retinoids on AOM-induced colon cancer in rats: modulation of cell proliferation, apoptosis and aberrant crypt foci. Carcinogenesis 20: 255-260, 1999.

11. Yamada Y, Yoshimi N, Hirose Y, et al: Frequent beta-catenin gene mutations and accumulations of the protein in the putative preneoplastic lesions lacking macroscopic aberrant crypt foci appearance, in rat colon carcinogenesis. Cancer Res 60: 3323-3327, 2000.

12. Yamada Y, Yoshimi N, Hirose Y, et al: Sequential analysis of morphological and biological properties of beta-cateninaccumulated crypts, provable premalignant lesions independent of aberrant crypt foci in rat colon carcinogenesis. Cancer Res 61: 1874-1878, 2001.

13. Femia AP, Dolara P and Caderni G: Mucin-depleted foci (MDF) in the colon of rats treated with azoxymethane (AOM) are useful biomarkers for colon carcinogenesis. Carcinogenesis 25: 277-281, 2004.

14. Femia AP, Giannini A, Fazi M, et al: Identification of mucin depleted foci in the human colon. Cancer Prev Res (Phila) 1: 562-567, 2008.

15. Yoshimi N, Morioka T, Kinjo T, et al: Histological and immunohistochemical observations of mucin-depleted foci (MDF) stained with Alcian blue, in rat colon carcinogenesis induced with 1,2-dimethylhydrazine dihydrochloride. Cancer Sci 95: 792-797, 2004.

16. Schlemper RJ, Riddell RH, Kato Y, et al: The Vienna classification of gastrointestinal epithelial neoplasia. Gut 47: 251-255, 2000.

17. Rectum JSfCotCa: General Rules for clinical and Pathological Studies on Cancer of the Colon, Rectum and Anus. 7th edition, Revised Version. Kanehara Shuppan, Ltd., Tokyo, 2009.

18. Bosman FT, Carneiro F, Hruban RH and Theise ND: WHO Classification of Tumors of the Digestive System. International Agency for Research on Cancer, Lyon, 2010.

19. Ward JM: Morphogenesis of chemically induced neoplasms of the colon and small intestine in rats. Lab Invest 30: 505-513, 1974.

20. Femia AP, Bendinelli B, Giannini A, et al: Mucin-depleted foci have beta-catenin gene mutations, altered expression of its protein, and are dose- and time-dependent in the colon of 1,2-dimethylhydrazine-treated rats. Int J Cancer 116: 9-15, 2005. 
21. Gendler SJ and Spicer AP: Epithelial mucin genes. Annu Rev Physiol 57: 607-634, 1995.

22. Kim YS, Gum J Jr and Brockhausen I: Mucin glycoproteins in neoplasia. Glycoconj J 13: 693-707, 1996.

23. Zusman I, Zimber A and Nyska A: Role of morphological methods in the analysis of chemically induced colon cancer in rats. Acta Anat (Basel) 142: 351-356, 1991.

24. Dawson PA, Patel J and Filipe MI: Variations in sialomucins in the mucosa of the large intestine in malignancy: a quantimet and statistical analysis. Histochem J 10: 559-572, 1978.

25. Filipe MI: Value of histochemical reactions for mucosubstances in the diagnosis of certain pathological conditions of the colon and rectum. Gut 10: 577-586, 1969.

26. Matsushita Y, Yamamoto N, Shirahama H, et al: Expression of sulfomucins in normal mucosae, colorectal adenocarcinomas, and metastases. Jpn J Cancer Res 86: 1060-1067, 1995.

27. Sandforth F, Heimpel S, Balzer T, Gutschmidt S and Riecken EO Characterization of stereomicroscopically identified preneoplastic lesions during dimethylhydrazine-induced colonic carcinogenesis. Eur J Clin Invest 18: 655-662, 1988.

28. Ochiai M, Ushigome M, Fujiwara K, et al: Characterization of dysplastic aberrant crypt foci in the rat colon induced by 2-amino-1-methyl-6-phenylimidazo[4,5-b]pyridine. Am J Pathol 163: 1607-1614, 2003.
29. Paulsen JE, Namork E, Steffensen IL, Eide TJ and Alexander J: Identification and quantification of aberrant crypt foci in the colon of Min mice - a murine model of familial adenomatous polyposis. Scand J Gastroenterol 35: 534-539, 2000.

30. Paulsen JE, Knutsen H, Olstorn HB, Loberg EM and Alexander J: Identification of flat dysplastic aberrant crypt foci in the colon of azoxymethane-treated A/J mice. Int J Cancer 118: 540-546, 2006.

31. Femia AP, Paulsen JE, Dolara P, Alexander J and Caderni G: Correspondence between flat aberrant crypt foci and mucindepleted foci in rodent colon carcinogenesis. Anticancer Res 28: 3771-3775, 2008

32. Ochiai M, Watanabe M, Nakanishi M, Taguchi A, Sugimura T and Nakagama H: Differential staining of dysplastic aberrant crypt foci in the colon facilitates prediction of carcinogenic potentials of chemicals in rats. Cancer Lett 220: 67-74, 2005

33. Schlemper RJ, Itabashi M, Kato Y, et al: Differences in the diagnostic criteria used by Japanese and Western pathologists to diagnose colorectal carcinoma. Cancer 82: 60-69, 1998. 